\title{
A THEORETICAL MODEL OF RADIATIVE TRANSFER IN YOUNG SEA ICE*
}

\author{
By Donald K. Perovich and Thomas C. Grenfell \\ (Department of Atmospheric Sciences AK-40, University of Washington, Seattle, Washington \\ 98195, U.S.A.)
}

\begin{abstract}
A four-stream discrete-ordinates photometric model including both anisotropic scattering and refraction at the boundaries is presented which treats the case of a floating ice slab. The effects of refraction and reflection on the redistribution of the incident radiation field as it enters the ice are examined in detail. Using oneand two-layer models, theoretical albedos and transmittances are compared to values measured in the laboratory for thin salt ice. With an experimentally determined three-parameter Henyey-Greenstein phase function, comparisons at $650 \mathrm{~nm}$ yield single-scattering albedos ranging from 0.95 to 0.9997 . The models are then used to compare the effects of diffuse and direct-beam incident radiation, to investigate the dependence of spectral albedo and transmittance on ice thickness, and to determine the influence of very cold and melted surface layers.
\end{abstract}

RÉSUMÉ. Un modèle théorique de transfert radiatif dans la jeune glace de mer. On présente un modèle photométrique à quatre flux pour des ordonnées discretes comprenant à la fois un balayage anisotropique et une réfraction aux limites qui traite le cas d'une plaque de glace flottante. Les effets de la réfraction et de la réflexion sur la redistribution de la radiation incidente lorsqu'elle pénètre dans la glace sont examinés en détail. En utilisant un ou deux niveaux modèles, les albédos théoriques et les transmittances sont comparées aux valeurs mesurées en laboratoire pour une fine glace salée. Avec une fonction de phase Henyey-Greenstein à trois paramètres déterminés expérimentalement, les comparaisons à $650 \mathrm{~nm}$ donnent des albédos à répartition unique allant de 0,95 à 0,999 7. Les modèles sont alors utilisés pour comparer les effets d'un éclair de radiation incidente directe et diffuse, et pour rechercher si l'albédo spectral et la transmittance dépendent de l'épaisseur de la glace, et pour déterminer l'influence des niveaux de surface très froids et en fusion.

ZuSAMmenfassung. Ein theoretisches Modell zur Strahlungsübertragung in frischem Meereis. Es wird ein photometrisches Vier-Strom-Modell mit diskreten Ordinaten, das sowohl anisotrope Streuung wie Refraktion an den Korngrenzen berücksichtigt, vorgelegt, das sich auf den Fall einer treibenden Eisscholle bezieht. Der Einfluss von Refraktion und Reflexion auf die Umverteilung des Feldes einfallender Strahlung, wenn sie in das Eis eindringt, wird im einzelnen untersucht. Mit Hilfe von Ein- und Zweischichtenmodellen werden theoretische Werte der Albedo und der Durchlässigkeit mit Messungen verglichen, die sich im Labor für dünnes salzhaltiges Eis ergaben. Mit Hilfe einer experimentell bestimmten Drei-Parameter-Phasenfunktion nach Henyey-Greenstein liefern Vergleiche bei $650 \mathrm{~nm}$ einzelne Streualbeden zwischen 0,95 und 0,999 7. Die Modelle werden dann herangezogen, um die Auswirkungen diffus bzw. gerichtet einfallender Strahlung zu vergleichen, um die Abhängigkeit der spektralen Albedo und Durchlässigkeit von der Eisdicke zu untersuchen und um den Einfluss sehr kalter bzw. aufgeschmolzener Oberflächenschichten zu bestimmen.

\section{INTRODUCTION}

Knowledge of the transfer of solar radiation in sea ice is intrinsic to determining energy balances in the polar regions. The reflection, absorption, and transmission of solar radiation affects both the heat and mass balance of the ice cover and the biological processes occurring under the ice cover. Sea ice is an optically complex medium dominated by scattering with an intricate physical structure consisting of air bubbles, ice platelets, brine pockets and channels, and crystals of various sizes and orientations. Because of this, the optical properties of the ice show strong temperature and seasonal dependencies. Simple photometric models used in the past describe the radiative processes occurring in sea ice only for limited cases where observational

* Department of Atmospheric Sciences, University of Washington. Contribution No. 621. 
data are available and cannot be generalized to different ice conditions. The Bouguer-Lambert law has been the most commonly used model, and although its simplicity is appealing, the assumption of a semi-infinite medium and simplified representations of volume scattering severely limit its effectiveness. A two-stream photometric model developed by Dunkle and Bevans (1956) to study a snow cover of finite thickness was adapted by Grenfell and Maykut (1977) to calculate albedos and extinction coefficients for both snow and sea ice. This model still assumed diffuse incident radiation (cloudy skies) and isotropic scattering; however, studies by T. C. Grenfell and D. Hedrick (personal communication) show that light scattering by sea ice is strongly anisotropic. In addition, the theory should be extended to include field distributions of incident radiation applicable for clear sky conditions.

The recent experimental results of Perovich (1979) and Perovich and Grenfell (1981) can be used to formulate and test more sophisticated models. These consist of laboratory observations of young sea ice including spectral albedo and transmission data for a significant variety of ice types under controlled environmental conditions along with concurrent measurements of temperature, salinity, and crystal structure.

The primary goal of the present work is to develop a photometric model that is consistent with the above-mentioned observations and avoids the limitations of earlier models while maintaining computational simplicity. To accomplish this, a four-stream discrete-ordinates model based on the work of Chandrasekhar (1950) and Liou (1973, 1974) was adapted to the special situation of a thin floating ice slab.

\section{RADIATIVE TRANSFER THEORY}

\section{Background}

The discrete ordinates method of Chandrasekhar (1950) gives solutions of any desired degree of accuracy to the equation of radiative transfer for a plane parallel, homogeneous, multiplescattering medium. The discrete ordinates, or streams, refer to the angles at which radiances are

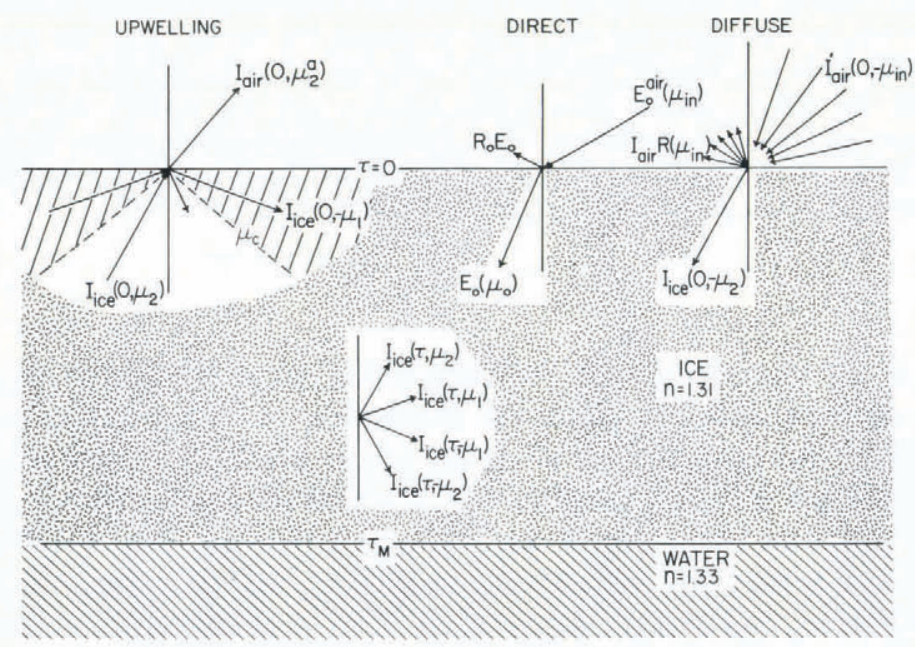

Fig. 1. Schematic representation of the four-stream model for a floating ice slab including refraction and specular reflection at the upper boundary. 
determined. Including more streams gives greater accuracy but also gives a greater degree of computational complexity. From Chandrasekhar (1950) the equation of radiative transfer for a plane parallel medium is

$$
\mu \frac{\mathrm{d} I(\tau, \mu, \phi)}{\mathrm{d} \tau}=I(\tau, \mu, \phi)-S(\tau, \mu, \phi)
$$

where $I$ is the radiance, $S$ is the source function, $\phi$ is the azimuth angle, and $\mu$ is the cosine of the zenith angle $\theta$. The optical depth $\tau$ is defined in terms of the volume scattering coefficient $\sigma$ and the absorption coefficient $k$ by

$$
\tau \equiv-(k+\sigma) z .
$$

The minus sign results from the choice that positive $\mu$ s represent upward angles. For the case of sea ice at visible wavelengths, thermal emission is negligible and $S$ describes the contribution of multiple scattering. For a plane parallel scattering atmosphere with a direct incident beam

$$
S=\frac{1}{4 \pi} \int_{-1}^{1} \int_{0}^{2 \pi} p\left(\mu, \phi ; \mu^{\prime}, \phi^{\prime}\right) I\left(\tau, \mu^{\prime}, \phi^{\prime}\right) \mathrm{d} \mu^{\prime} \mathrm{d} \phi^{\prime}+\frac{E_{0}}{4} p\left(\mu, \phi ; \mu_{0}, \phi_{0}\right) \mathrm{e}^{-\tau / \mu_{0}} .
$$

Axial symmetry holds for the experimental results under study and Equation (1) gives

$$
\mu \frac{\mathrm{d} I(\tau, \mu)}{\mathrm{d} \tau}=I(\tau, \mu)-\frac{1}{2} \int_{-1}^{1} p\left(\mu, \mu^{\prime}\right) I\left(\tau, \mu^{\prime}\right) \mathrm{d} \mu^{\prime}-\frac{E_{0}}{4} p\left(\mu, \mu_{0}\right) \mathrm{e}^{-\tau / \mu_{0}}
$$

where $E_{0}$ is the direct solar irradiance and $\mu_{0}$ is the absolute magnitude of the cosine of the solar zenith angle.

The scattering processes within the medium are jointly defined by the phase function $p\left(\mu, \mu^{\prime}\right)$ and the single scattering albedo $\varpi_{0}$. The phase function describes the angular dependence of scattering and typically is normalized so that its integral over solid angle is equal to one. For use in the discrete ordinates method, it is expressed in terms of the following expansion in Legendre polynomials,

$$
p\left(\mu, \mu^{\prime}\right)=\sum_{l=0}^{N-1} \varpi_{l} P_{l}(\mu) P_{l}\left(\mu^{\prime}\right)
$$

where $N$ is the number of streams. The relative values of the $\varpi_{l} s$ in Equation (3) are obtained for a particular phase function from

$$
\varpi_{l}=\frac{2 l+1}{2} \int_{-1}^{1} p\left(1, \mu^{\prime}\right) P_{l}\left(\mu^{\prime}\right) \mathrm{d} \mu^{\prime}
$$

which is derived by exploiting the orthogonality of the Legendre polynomials. These values must then be multiplied by the single-scattering albedo which describes the relative importance of scattering and absorbing processes and is defined as

$$
\varpi_{0}=\frac{\sigma}{\sigma+k}
$$

and ranges from zero for a purely absorbing medium to one for a purely scattering medium.

Liou $(1973,1974)$ adapted Chandrasekhar's general solution to investigate radiative transfer 
within cloudy and hazy atmospheres. Analytic solutions were determined for the two- and fourstream cases with numerical solutions for eight and sixteen streams. In two-stream models the errors became prohibitively large for optically thin layers. The eight- and sixteen-stream models, while offering greater angular resolution of radiance and a more precise representation of complex phase functions, required a complicated iterative solution scheme. Thus, the four-stream case was chosen to take advantage of the mathematical convenience while retaining sufficient accuracy (Liou, 1974). For this model, Liou (1974) gives the solutions for the upwelling and downwelling irradiances in terms of a four-point Gaussian quadrature as follows:

$$
F \uparrow(\tau)=2 \pi\left[a_{1} \mu_{1} I\left(\tau, \mu_{1}\right)+a_{2} \mu_{2} I\left(\tau, \mu_{2}\right)\right]
$$

and

$$
F \downarrow(\tau)=-2 \pi\left[a_{1} \mu_{1} I\left(\tau,-\mu_{1}\right)+a_{2} \mu_{2} I\left(\tau,-\mu_{2}\right)\right]-\pi \mu_{0} E_{0} \mathrm{e}^{-\tau / \mu_{0}}
$$

where $I(\tau, \pm \mu)$ is the radiance in each of the streams, the $a_{j}$ s are the Gaussian weighting functions $\left(a_{1}=0.6521452\right.$ and $\left.a_{2}=0.3478548\right)$, and the $\mu$ s are the cosines of the zenith angles for each of the four streams $\left(\mu_{1}=0.3399810\right.$ and $\left.\mu_{2}=0.8611363\right)$.

\section{Application to sea ice}

In general the ice structure is assumed to be homogeneous and is represented by a singlelayer model. It is also necessary to take into account that the medium is a collection of scattering centers embedded in ice rather than in air. Thus, refraction and specular reflection modify the incident radiation field as it enters the ice. To illustrate the importance of refraction and reflection, solutions are carried out both ignoring and including these effects for simple cases.

\section{No refraction}

In this case the boundary conditions are

$$
\left.I\left(0,-\mu_{1}\right)=I\left(0,-\mu_{2}\right)=C \text { (upper boundary }\right)
$$

and

$$
I\left(\tau_{\mathrm{M}}, \mu_{1}\right)=I\left(\tau_{\mathrm{M}}, \mu_{2}\right)=0 \text { (lower boundary) }
$$

where $\tau_{M}$ is the optical thickness of the ice slab. For isotropic incident radiation representing cloudy sky conditions, $C$ is equal to one and there is no direct beam component $\left(E_{0}=0\right)$. To represent clear skies, $C$ is assumed to be zero and the incident radiation field is defined by a direct beam of radiance $E_{0}$ at an angle $\arccos \left(\mu_{0}\right)$. The lower boundary condition is set by assuming that the underlying water does not scatter radiation back into the ice.

The albedo and transmittance are determined from $\alpha=F \uparrow(0) / F_{0}$ and $T=F \downarrow\left(\tau_{\mathrm{M}}\right) / F_{0} . F_{0}$ is the incident irradiance and is equal to $\pi$ for isotropic incident radiation and $\mu_{0} \pi E_{0}$ for an incident direct beam. Equations (6) and (7) are used to calculate $F \uparrow(0)$ and $F \downarrow\left(\tau_{\mathbf{M}}\right)$.

\section{Refraction}

At the ice-air boundary, refraction must be considered in detail. From geometric considerations (Jerlov, 1976) or variable transformation theory (Papoulis, 1965), the redistribution of a diffuse incident radiation field by refraction is given by

$$
I_{\text {ice }}(0,-\mu)=n^{2}\left[1-R\left(-\mu^{\mathrm{a}}\right)\right] I_{\text {air }}\left(0,-\mu^{\mathrm{a}}\right)
$$


where $R\left(-\mu^{\mathrm{a}}\right)$ is the Fresnel reflection coefficient for natural light and $\mu^{\mathrm{a}}$ and $\mu$ are corresponding direction cosines in the air and the ice respectively. For isotropic incident radiation $I_{\text {air }}\left(0,-\mu^{\mathrm{a}}\right)=1$ and $\mu^{\mathrm{a}}$ ranges from -1 to 0 , but $\mu$ extends from only -1 to -0.646 . Figure 2 displays the redistribution of this radiation field at the ice-air interface. Before refraction, the radiance is one at all angles. Specular reflection removes much of the radiation near grazing incidence, and refraction concentrates the remaining downwelling radiance between 0 and $49.8^{\circ}$.

After refraction, $I_{\text {ice }}\left(0,-\mu_{2}\right)=1.67$ while $I_{\text {ice }}\left(0,-\mu_{1}\right)=0$. As a result the quadrature in Equation (7) has only one non-zero term remaining which introduces considerable inaccuracy in the calculation of downwelling irradiance. In order to conserve energy, the value of $I_{\text {ice }}\left(0,-\mu_{2}\right)$ is adjusted to make Equation (5) consistent with the formula (Chandrasekhar, 1950).

$$
F \downarrow_{\text {ice }}(0)=2 \pi \int_{-1.0}^{-0.646} \mu I_{\text {ice }}(0, \mu) \mathrm{d} \mu .
$$

Numerical integration gives $F \downarrow_{\text {ice }}(0)=0.937 \pi$ so that $I_{\text {ice }}\left(0,-\mu_{2}\right)=1.56$ which is used in Equation (7) in place of 1.67.

The upwelling radiance at $\tau=0$ is similarly influenced by refraction. None of the radiation striking the boundary at angles exceeding the critical angle for total internal reflection $\left(\theta_{\mathrm{c}}=49.8^{\circ}\right)$ emerges from the ice, including $I_{\text {ice }}\left(0, \mu_{1}\right)$. In addition, $2.2 \%$ of $I_{\text {ice }}\left(0, \mu_{2}\right)$ is reflected downward by the interface. For diffuse incident radiation then, the upper boundary conditions are

$$
I_{\text {ice }}\left(0,-\mu_{1}\right)=I_{\text {ice }}\left(0,+\mu_{1}\right)
$$

and

$$
I_{\text {ice }}\left(0,-\mu_{2}\right)=0.022 I_{\text {ice }}\left(0,+\mu_{2}\right)+1.56 \text {. }
$$

If the incident radiation field is a direct beam, $E_{0}\left(\mu_{\text {in }}\right)$, the magnitude of the refracted beam is

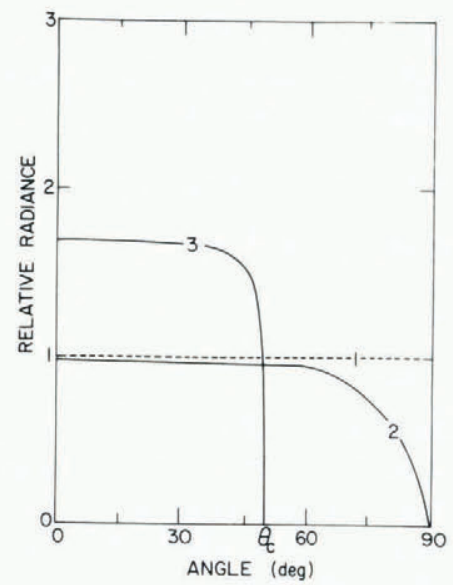

Fig. 2. The angular distribution of isotropic incident radiation undergoing refraction: (1) isotropic incident radiation; (2) net incident radiation; and (3) radiation distribution in the ice after refraction $\left(\theta=49.5^{\circ}\right.$ is the angle of total internal reflection). 
given by

$$
E_{0}\left(\mu_{0}\right)=E_{0}\left(\mu_{\text {in }}\right)\left[1-R\left(\mu_{\text {in }}\right)\right] \frac{\mu_{\text {in }}}{\mu_{0}}
$$

where $\mu_{\text {in }}$ is the cosine of the zenith angle of the direct incident beam in air and $\mu_{0}$ is cosine of the corresponding angle after refraction (Born and Wolf, 1964). Since this beam is included explicitly in the equation of transfer as a volume source term, the boundary conditions are the same as for the diffuse case except that the 1.56 does not appear in Equation (9).

At the bottom interface, the small difference in the refractive indices of ice and water gives a very small amount of specular reflection. For example, only $0.26 \%$ and $0.01 \%$ of the radiation reaching the lower boundary at angles $-\mu_{1}$ and $-\mu_{2}$ is reflected back into the ice. Consequently, the effect can be ignored, and the lower boundary conditions remain the same as for the no-refraction case.

The formula for total transmittance retains the same form, $T=F \downarrow\left(\tau_{\mathrm{M}}\right) / F_{0}$, but calculating the albedo is more involved. The general expression for albedo is

$$
\alpha=\frac{2 \pi \int_{0}^{1} \mu_{\text {in }} I_{\text {air }}\left(0,-\mu_{\text {in }}\right) R\left(\mu_{\text {in }}\right) \mathrm{d} \mu_{\text {in }}+2 \pi \int_{0.696}^{1} \mu I_{\text {ice }}(\mu)[1-R(\mu)] \mathrm{d} \mu}{F_{0}} .
$$

For the direct-beam case, the first integral in the numerator is equal to $\pi \mu_{\text {in }} R\left(\mu_{\text {in }}\right) E_{0}\left(\mu_{\text {in }}\right)$. For diffuse incidence, numerical integration gives a value of $0.065 \pi$. The second integral would normally be evaluated by Gaussian quadrature, but because $I\left(0, \mu_{1}\right)$ is totally reflected, only one non-zero term would be present with a resulting loss in accuracy. Therefore the upwelling radiance is fitted to a function of the form $I_{\text {ice }}(\mu)=A \mu+B$ (as is the Eddington assumption (Joseph and others, 1976)), where $A=\left[I_{\text {ice }}\left(0, \mu_{1}\right)-I_{\text {ice }}\left(0, \mu_{2}\right)\right] /\left(\mu_{1}-\mu_{2}\right)$ and $B=I_{\text {ice }}\left(0, \mu_{1}\right)-$ $\mu_{1} A$. This allows the value of $I_{\text {ice }}\left(0, \mu_{1}\right)$ to contribute to $\alpha$. Then by assuming $R(\mu) \approx 0.065$, the second integral in the numerator of Equation (10) can be evaluated analytically giving

$$
\alpha=R_{0}+0.454 A+0.544 B
$$

where $R_{0}=0.065$ for isotropic incident radiance and $R\left(\mu_{\text {in }}\right)$ for a direct incident beam.

In some cases, a two-layer model is needed. To extend the model, four additional boundary conditions must be satisfied at the interface. These are given by

$$
I_{\text {layer } 1}\left(\tau_{\text {bdy }}, \pm \mu_{1,2}\right)=I_{\text {layer } 2}\left(\tau_{\text {bdy }}, \pm \mu_{1,2}\right)
$$

where the index of refraction is the same in both layers.

\section{Model parameters}

To complete the model, the following quantities are specified: the phase function, the absorption coefficient, the ice thickness, and either the scattering coefficient or the singlescattering albedo. The total optical depth and $\varpi_{0}$ or $\sigma$ are then given by Equations (2) and (5).

The ice thickness is measured directly, and absorption coefficients for ice are taken from the results of Sauberer (1950) as reported by Goodrich (1970). For the phase function of sea ice, the experimental data of T. C. Grenfell and D. Hedrick (personal communication) are used. Curve 1 in Figure 3 is a smoothed fit of their preliminary results. A large backscattering component is present compared with that due to air bubbles in water (Davis, 1955) or ice. For mathematical convenience, a Henyey-Greenstein phase function (Henyey and Greenstein, 1941) is used to 


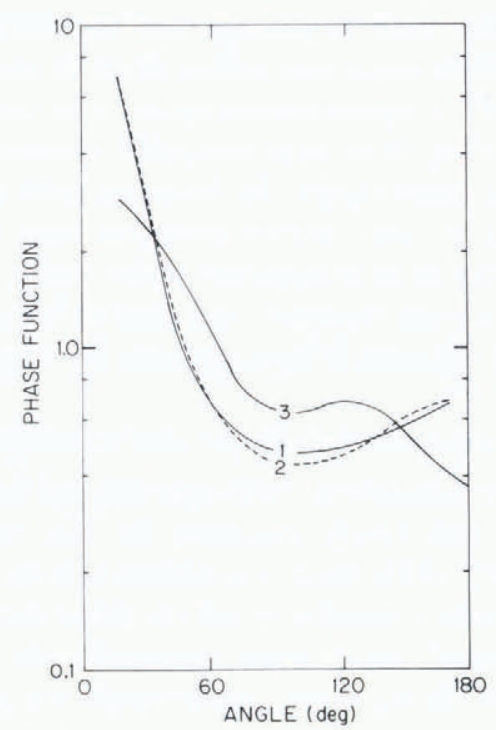

Fig. 3. Representations of the phase function used for young sea ice. Curve $I$ is the experimental results of Grenfell and Hedrick. Curve 2 is the best fit of curve 1 using a three-parameter Henyey-Greenstein phase function. Curve 3 displays the four-stream approximation to curve 2.

represent $p(\Theta)$. To include the backscattering component, a three-parameter function of the form

$$
p(\Theta)=(1-\gamma) \frac{1-g_{1}^{2}}{\left(1+g_{1}^{2}-2 g_{1} \cos \Theta\right)^{3 / 2}}+\gamma \frac{1-g_{2}^{2}}{\left(1+g_{2}^{2}-g_{2} \cos \Theta\right)^{3 / 2}}
$$

is adopted, where the second term contributes the backscattering $\left(g_{2}<0\right)$, and $\gamma$ is the relative weighting factor between two terms. A non-linear regression analysis was used to determine the coefficients. Best-fit values were found to be $\gamma=0.279, g_{1}=0.71$, and $g_{2}=-0.25$, and the resulting phase function is plotted as curve 2 in Figure 3. To show how accurately $p(\Theta)$ is represented by the four-term expansion, the associated $\varpi_{l} \mathrm{~s}$ were determined from Equation (4) by numerical integration and substituted into Equation (3). This derived phase function, curve 3 in Figure 3, deviates from the shape of the original curve most strongly at large scattering angles. But since most of the scattering in the four-stream model involves deflections of $120^{\circ}$ or less, the approximation is quite good, and errors in the irradiance are not large (Liou, 1974).

\section{Calculations}

\section{Response of the model to variations in the basic parameters}

A range of cases was examined to investigate the effect of the single-scattering albedo and the phase function on the calculated albedo and transmittance. For a particular ice thickness, increasing $\varpi_{0}$ gives an increase in albedo and a decrease in transmittance. As $\varpi_{0}$ approaches one, albedo and transmittance become very sensitive to small changes in $\varpi_{0}$. Calculations for a variety of phase functions indicate that albedo decreases and transmittance increases as $p(\Theta)$ becomes more forward-peaked. 
Albedos and transmittances, both neglecting and considering refraction at the air-ice interface, were calculated for direct-beam and diffuse incident radiation. The zenith angle of the direct beam was taken to be $65^{\circ}$, a representative value for Arctic latitudes in the summer. The results are given in Table I. They show that refraction causes a decrease in albedo and an increase in transmittance. This results from the following effects: A portion of the upwelling radiance at the surface undergoes total internal reflection back into the ice, and much of this radiation is then absorbed by the ice or transmitted to the water. Also, refraction converges the incident radiation into a more normal direction, and, because the scattering is primarily forwarddirected, a larger fraction of the radiation is transmitted to the water.

\section{Comparison of theoretical and experimental albedos and transmittances}

Comparisons of four-stream calculations with experimental results were performed primarily at $650 \mathrm{~nm}$. This wavelength was nearly optimum for the spectrophotometer used in the observations (Roulet and others, 1974) since it combines a large signal-to-noise ratio with high spectral resolution. This also made it possible to use the phase functions of T. C. Grenfell and D. Hedrick (personal communication), which were measured with a helium neon laser. Since an optical diffuser was used during the laboratory experiments, an isotropic incident radiation field was assumed for all calculations pertaining to the observational results.

For a particular experimental case, $\varpi_{0}$ was selected so that the albedo calculated using the four-stream model would best match the experimental albedo. In order to compensate for the effects of the spectrophotometer's bandwidth, a corrected absorption coefficient of $0.42 \mathrm{~m}^{-1}$ was used rather than the standard value at $650 \mathrm{~nm}$ of $0.32 \mathrm{~m}^{-1}$ (Sauberer, 1950). The corrected value was determined by convolving a representative spectral transmission curve with the bandpass of the instrument.

This technique was applied to data from ten experimental cases and the results summarized in Table II. Albedos for cases 3 through 10 were matched with values of $\varpi_{0}$ ranging from 0.955 to 0.988 . The difference between theoretical and experimental transmittances ranges from 0.6 to $41 \%$ and in all cases but one, the theoretical transmittance is larger than the experimental. The differences are smallest for warm, slowly grown ice and largest for the very cold, rapidly grown ice. Best agreement is achieved for cases 7,8 , and 10 , where the ice was most nearly homogeneous.

TABLE I. EFFECTS OF REFRACTION ON ALBEDO AND TRANSMITTANCE

$\begin{aligned} & \text { Ice thickness } \\ & \quad 25 \mathrm{~cm}\end{aligned}$
$\varpi_{0}=0.97, \tau_{\mathrm{M}}=2.67$
Albedo
Transmittance
$\varpi_{0}=0.98, \tau_{\mathrm{M}}=4.0$
Albedo
Transmittance
$\varpi_{0}=0.99, \tau_{\mathrm{M}}=8.0$
Albedo
Transmittance

\begin{tabular}{|c|c|c|c|c|c|}
\hline \multicolumn{3}{|c|}{ Diffuse incident radiation } & \multicolumn{3}{|c|}{ Direct beam $\mu_{\text {in }}=0.423\left(\theta=65^{\circ}\right)$} \\
\hline No & & $\%$ & No & & $\%$ \\
\hline refraction & Refraction & difference & refraction & Refraction & difference \\
\hline 0.534 & 0.362 & -32.2 & 0.599 & 0.397 & -33.7 \\
\hline 0.330 & 0.449 & 36.1 & 0.264 & 0.409 & 54.9 \\
\hline 0.619 & 0.447 & -27.8 & 0.674 & 0.479 & -28.9 \\
\hline 0.247 & 0.347 & 40.5 & 0.199 & 0.317 & 59.3 \\
\hline 0.739 & 0.581 & -21.4 & 0.778 & 0.605 & -22.2 \\
\hline 0.133 & 0.197 & 48.1 & 0.108 & 0.181 & 67.6 \\
\hline
\end{tabular}




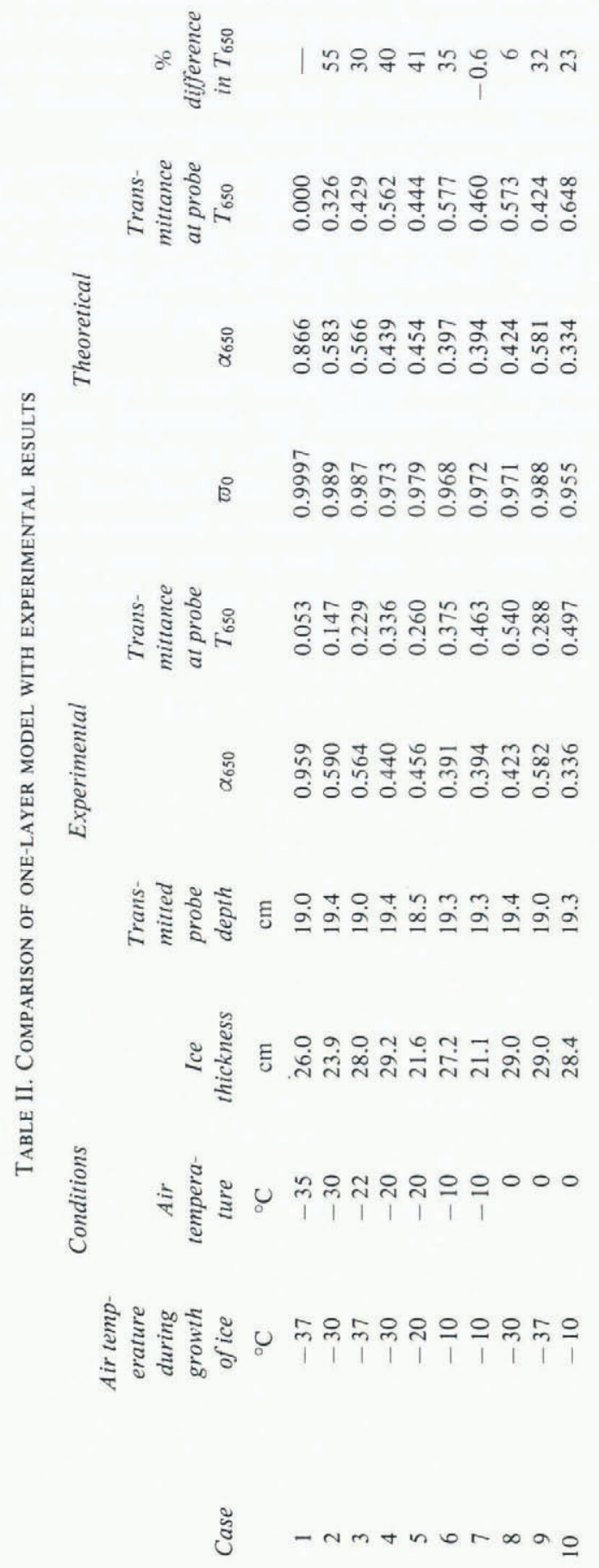


The assumption of homogeneous ice is probably the largest source of error in the theoretical calculations. Temperature gradients, changes in growth rate with ice thickness, and differences in crystal structure all cause vertical variations in the physical state of the ice which can affect the optical properties. In addition, changes in the ice surface strongly influence the albedo and transmittance. This will be discussed in detail in the next section.

For cases 1 and 2, which were most poorly represented by the one-layer analysis, a two-layer model was applied. These cases had the largest temperature gradients, and the surface temperature of the ice was at or below the eutectic point. In case 1, for example, the top two centimeters of ice were below the eutectic point for $\mathrm{NaCl}$. This resulted in a region containing precipitated salts whose optical properties were distinctly different from those of the underlying ice.

The two-layer calculations which best represent cases 1 and 2 are summarized in Table III. While the agreement between experimental and theoretical results is improved, substantial differences still remain suggesting that for cold ice a multilayer model may be necessary. In addition, for case 1 it was not possible to fit the albedo precisely. This was probably due to the presence of patches of highly scattering $\mathrm{NaCl}$ crystals distributed unevenly across the surface of the ice. By assuming refraction for these patches their enhancement of the albedo is underestimated. To match the observed albedo, considerable reformulation of the model would be needed. However, a more detailed analysis of the experimental results is not warranted until more comprehensive data concerning the relationships of the single-scattering albedo and the phase function to the state and structure of the ice are available.

For several cases spectral albedos and transmittances were calculated at $50 \mathrm{~nm}$ intervals between 400 and $1000 \mathrm{~nm}$. The spectral dependence of $k_{\lambda}$ for pure ice was determined from the results of Sauberer (1950) in the same fashion as $k_{650}$. Since scattering inhomogeneities in the ice are much larger than the wavelengths involved, the limit of geometrical optics applies for scattering by sea ice. Ellison and Peetz (1959) show that in this limit the wavelength dependence of scattering by spheres is determined by the index of refraction and is not influenced by particle size. This result also applies to nonspherical scatterers. Since the index of refraction is a weak function of wavelength from 400 to $1000 \mathrm{~nm}$, the scattering coefficient and the phase function are assumed to be independent of wavelength in this range.

Ice $21.1 \mathrm{~cm}$ thick grown at an air temperature of $-10^{\circ} \mathrm{C}$ (case 7) was selected as the most suitable case for comparison since the agreement at $650 \mathrm{~nm}$ was best. A scattering coefficient of $11.9 \mathrm{~m}^{-1}$ was determined from the value of $\varpi_{0}$ at $650 \mathrm{~nm}$ given in Table II. Theoretical spectral albedos are plotted together with the experimental values in Figure 4. The agreement between the two curves is quite good at all wavelengths; however, the slope of the theoretical curve is slightly greater, and calculated albedos lie above observed values from 400 to $650 \mathrm{~nm}$ and below them

TABLE III. COMPARISON OF TWO-LAYER MODEL WITH EXPERIMENTAL RESULTS FOR COLD ICE.

\begin{tabular}{|c|c|c|c|c|c|c|c|c|c|}
\hline \multirow[b]{2}{*}{ Case } & \multirow{2}{*}{$\begin{array}{c}\text { Air } \\
\text { tempera- } \\
\text { ture } \\
{ }^{\circ} \mathrm{C}\end{array}$} & \multicolumn{2}{|c|}{ Layer I } & \multicolumn{2}{|c|}{ Layer 2} & \multicolumn{2}{|c|}{ Theoretical } & \multicolumn{2}{|c|}{ Experimental } \\
\hline & & $\varpi_{0}$ & $\begin{array}{c}\text { Thickness } \\
\mathrm{cm}\end{array}$ & $\varpi_{0}$ & $\begin{array}{c}\text { Thickness } \\
\mathrm{cm}\end{array}$ & $\alpha_{650}$ & $T_{650}$ & $\alpha_{650}$ & $T_{650}$ \\
\hline 1 & -37 & 0.99993 & 2.0 & 0.97 & 24.0 & 0.898 & 0.018 & 0.959 & 0.053 \\
\hline 2 & -30 & 0.992 & 11.0 & 0.98 & 13.0 & 0.599 & 0.282 & 0.590 & 0.147 \\
\hline
\end{tabular}

$T_{650}$ is the transmittance at the probe. 


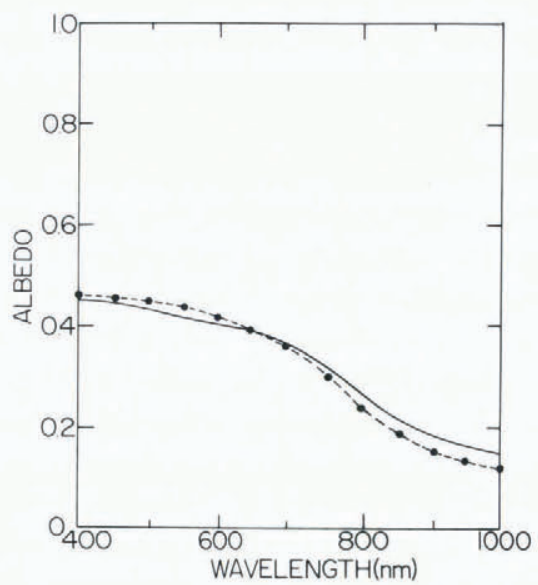

Fig. 4. Comparison of spectral albedos for $0.21 \mathrm{~m}$ thick ice at an air temperature of $-10^{\circ} \mathrm{C}:-$ experimental, -.-- theoretical. Theoretical calculations used $\sigma=11.9 \mathrm{~m}^{-1}$ and a diffuse incident radiation field.

from 650 to $1000 \mathrm{~nm}$. A comparison of the corresponding transmittances is shown in Figure 5. Due to the previously mentioned instrumental limitations, the observations are only given from 500 to $800 \mathrm{~nm}$. In this region the agreement with observations is acceptable, but again the theoretical curve drops off more rapidly with wavelength, crossing the observational curve at $650 \mathrm{~nm}$. The reason for this behavior is not clear at present, but may be due in part to uncertainties in the absorption coefficients of pure ice. The same analysis was performed for

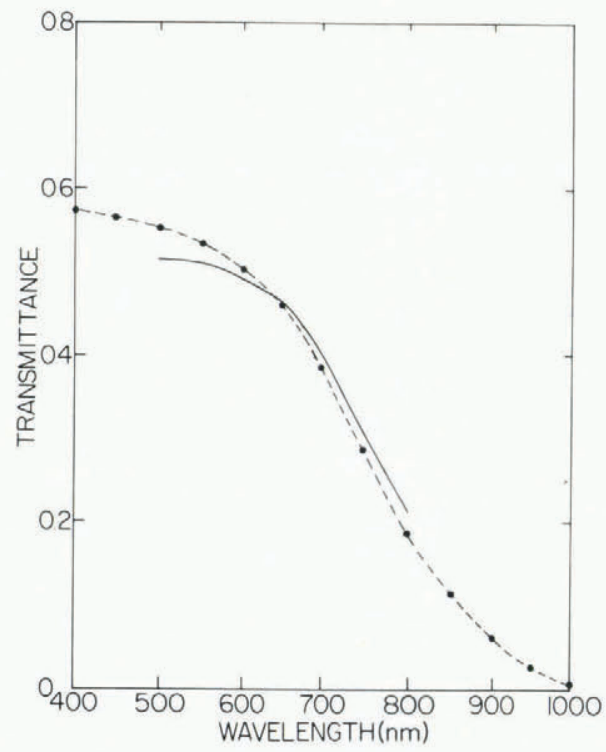

Fig. 5. Comparison of spectral transmittances for $0.21 \mathrm{~m}$ thick ice at an air temperature of $-10^{\circ} \mathrm{C}$ : experimental, -- - - theoretical. Theoretical calculations used $\sigma=11.9 \mathrm{~m}^{-1}$ and a diffuse incident radiation field. 
cases 8 and 10. As indicated in Table II, the absolute agreement was not as good, but the results show the same relative behavior for both albedos and transmittances.

\section{Further applications of the model}

The effect of the angle of incidence on albedo and transmittance of a $25 \mathrm{~cm}$ thick ice slab for clear sky conditions is investigated. Clear sky conditions are approximated by representing the incident radiation field as a direct beam at $\theta_{\text {in }}$. From 0 to $60^{\circ}$ albedo and transmittance vary gradually with zenith angle, but from $60^{\circ}$ to $90^{\circ}$ they are very sensitive to changes in $\theta_{\text {in }}$. This is a result of the increase in the Fresnel reflection of the direct beam and the forward peak of the phase function. For comparison $\alpha$ and $T$ were computed for cloudy skies. At approximately $54^{\circ}$, results for direct-beam incident radiation are equal to those for diffuse incident radiation in all three cases (Fig. 6).

The magnitude and spectral dependence of albedo and transmittance are markedly influenced by ice thickness. Using a representative scattering coefficient for growing ice at $-20^{\circ} \mathrm{C}$ of $15.7 \mathrm{~m}^{-1}$, spectral albedos and transmittances have been calculated for ice ranging in thickness from 5 to $25 \mathrm{~cm}$ and are shown in Figures 7 and 8 . As the ice thickens, the increase in albedo is greatest at shorter wavelengths where $\varpi_{0}$ is largest. Near $1000 \mathrm{~nm}$ no increase occurs for ice thicker than 5 to $7 \mathrm{~cm}$ since the ice is already optically thick. Radiation which penetrates below these depths and is then backscattered is almost completely absorbed before it can emerge from the ice. As the thickness approaches zero, the albedo approaches the limit of Fresnel reflection for water $(0.065)$. The transmittance is strongly dependent on wavelength, decreasing sharply in the infrared. As the ice thickness increases, the radiation near $1000 \mathrm{~nm}$ is quickly absorbed by the ice and when the ice is $15 \mathrm{~cm}$ thick, the transmission is essentially zero. In the visible the attenuation is much more gradual for $25 \mathrm{~cm}$ ice and the transmittance is reduced by only a factor of 2 .

During the laboratory experiments, the ice frequently developed a thin surface layer which caused a visible change in its appearance. Two important cases of this were encountered, one occurred when the temperature of the surface dropped below the eutectic point precipitating out solid salts within the ice, and the other when the ice was warm and water began to collect on the

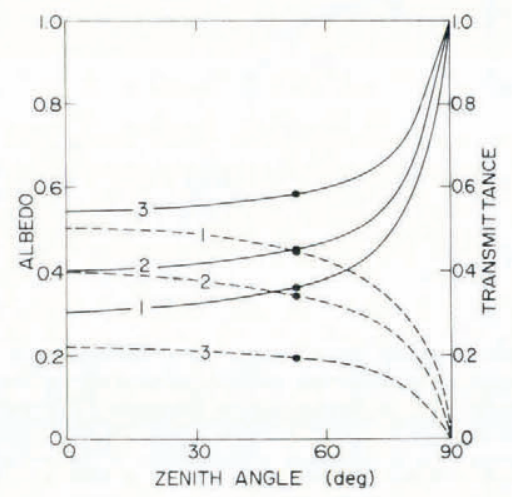

Fig. 6. Albedo _ and transmittance - - - as a function of the zenith angle of a direct incident beam for: (I) $\varpi_{0}=0.97,(2) \varpi_{0}=0.98$, and (3) $\varpi_{0}=0.99$. The values for isotropic incident radiation are given by the large dots, and are plotted on the curves to give the effective zenith angle. 


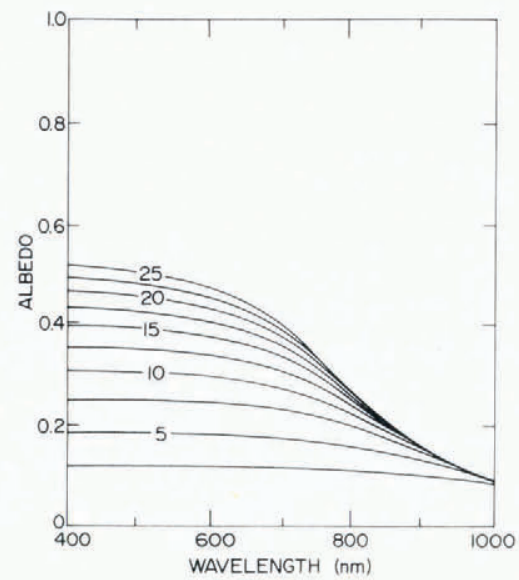

Fig. 7. Spectral albedos calculated for ice ranging in thickness from 0.025 to $0.25 \mathrm{~m}$ using a scattering coefficient of $15.7 \mathrm{~m}^{-1}$ for a diffuse incident radiation field. The curve labels indicate the ice thickness in centimeters.

surface. To give a quantitative estimate of the effects of these layers on the spectral albedo, twolayer models are constructed, again assuming $\sigma=15.7 \mathrm{~m}^{-1}$ for the underlying ice. In the surface layer, scattering coefficients of $400 \mathrm{~m}^{-1}$ and $6.8 \mathrm{~m}^{-1}$ are chosen based on cases 1 and 10 in Table II for very cold ice and melting ice respectively. Spectral albedos are then calculated for ice $25 \mathrm{~cm}$ thick including 1 and $5 \mathrm{~cm}$ surface layers of both ice types and for homogeneous ice without a surface layer. The results are plotted in Figure 9.

The presence of a cold and highly scattering layer increases the albedo considerably and alters its spectral distribution. For a $1 \mathrm{~cm}$ surface layer (curve 4 ) the increase is about $29 \%$ at $400 \mathrm{~nm}$ rising to $250 \%$ at $1000 \mathrm{~nm}$. The albedo is nearly constant across the visible region and the ice appears white. A $5 \mathrm{~cm}$ layer (curve 5) further increases the albedo at visible wavelengths by $25 \%$ almost uniformly from 400 to $650 \mathrm{~nm}$. In the infrared, however, the increase is smaller

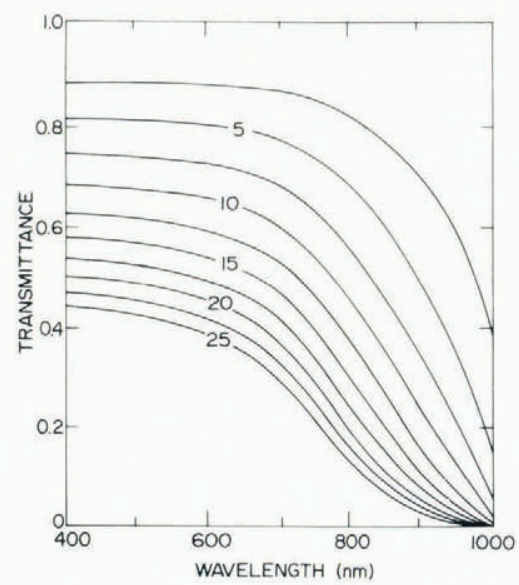

Fig. 8. Spectral transmittances calculated for ice ranging in thickness from 0.025 to $0.25 \mathrm{~m}$ using a scattering coefficient of $15.7 \mathrm{~m}^{-1}$ for diffuse incident radiation. The curve labels indicate the ice thickness in centimeters. 


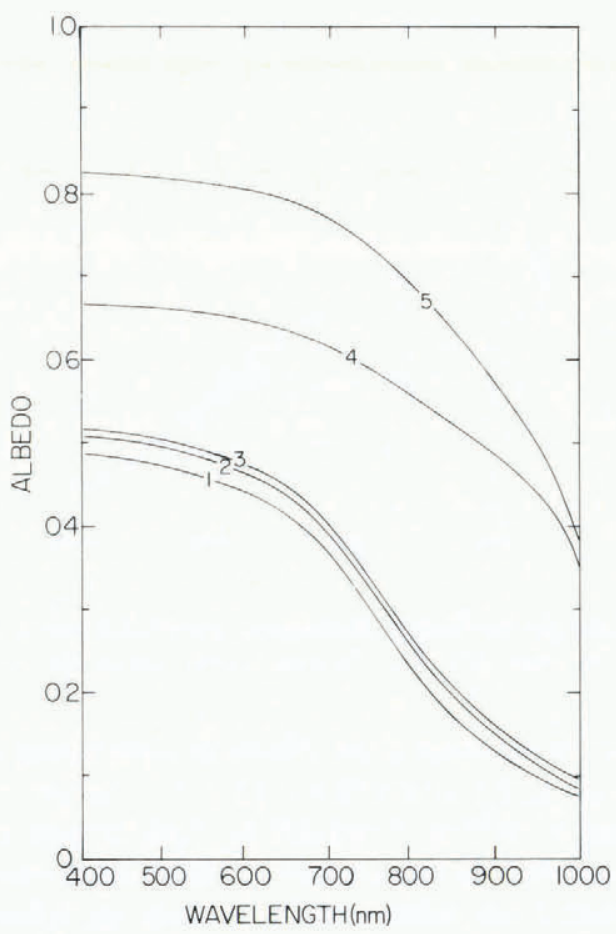

Fig. 9. Effects of different surface layers on spectral albedos for diffuse incident radiation. The total ice thickness is $0.25 \mathrm{~m}$ and the scattering coefficient for the underlying ice is $15.7 \mathrm{~m}^{-1}:$ (1) melting surface layer $50 \mathrm{~mm}$ thick $\left(\sigma=6.8 \mathrm{~m}^{-1}\right)$; (2) melting surface layer $10 \mathrm{~mm}$ thick $\left(\sigma=6.8 \mathrm{~m}^{-1}\right)$; (3) no surface layer; (4) highly scattering surface layer $10 \mathrm{~mm}$ thick $\left(\sigma=400 \mathrm{~m}^{-1}\right)$; and (5) highly scattering surface layer $50 \mathrm{~mm}$ thick $\left(\sigma=400 \mathrm{~m}^{-1}\right)$. Diffuse incident radiation was assumed for all calculations.

and at $1000 \mathrm{~nm}$ amounts to only about $8 \%$. This saturation effect at long wavelengths, also observed in Figure 7, arises because the thickness of ice contributing significantly to the albedo decreases as $k_{\lambda}$, and hence wavelength, increases.

The presence of a thin melting layer has a much smaller influence. A $1 \mathrm{~cm}$ layer decreases the albedo uniformly at all wavelengths (curve 2) by about 0.01 . Increasing the layer thickness from 1 to $5 \mathrm{~cm}$ (curve 1) further decreases $\alpha_{\lambda}$ by 0.025 but does not significantly alter the shape of the curve.

\section{Conclusions}

The four-stream model provides an effective method for examining the radiative processes in sea ice. It has added flexibility and accuracy over previous photometric models since it can account for anisotropic scattering and non-diffuse incident radiation. The effect of refraction at the surface is significant and is taken into account explicitly in the calculations. Modeling of the experimental results, using an empirical three-parameter Henyey-Greenstein phase function, indicates that $\varpi_{0}$ at $650 \mathrm{~nm}$ ranges from 0.955 for warm, slowly grown ice to as high as 0.99993 for ice below the eutectic point. Calculations of albedo and transmittance under clear 
sky conditions give the same results as diffuse incident radiation when the solar zenith angle is $54^{\circ}$. Theoretical and experimental albedos are matched at $650 \mathrm{~nm}$ by choosing an appropriate value of $\varpi_{0}$, and the resulting theoretical transmittances compared with observations. Agreement is good only for those cases where the ice was most homogeneous. For the coldest ice a twolayer model was introduced and improved the agreement somewhat, but it appears that a multilayer model is necessary. For the most homogeneous ice, theoretical calculations are extended over the wavelength interval 400 to $1000 \mathrm{~nm}$ and agree well with experimental results. Results from a two-layer model investigating effects of different types of surface layers show that a cold, highly scattering layer, such as ice below the eutectic point, causes an increase in the spectral albedo curve with layer thickness which is strongly wavelength dependent. For a warm melting layer on the other hand, the albedo decreases uniformly and more gradually.

Although multi-layer models are necessary to represent accurately most natural ice types, such efforts are severely limited by a scarcity of data concerning the scattering coefficients and the phase function. Experiments on small homogeneous ice samples are needed to determine single-scattering albedos and phase functions over an extensive range of temperature and structural conditions. Also needed are more accurate measurements of the spectral absorption coefficient for pure bubble-free ice, particularly at short wavelengths where the values are very small.

\section{ACKNOWLEDGEMENTS}

We would like to thank Dr Gary Maykut for his helpful assistance in preparing the text. This work was made possible by continued support from the Office of Naval Research, Arctic Program, under Contract N00014-76-C-0234.

MS. received 31 December 1980 and in revised form 13 May 1981

\section{REFERENCES}

Born, M., and Wolf, E. 1964. Principles of optics. Second revised edition. New York, Pergamon Press.

Chandrasekhar, S. 1950. Radiative transfer. Oxford, Clarendon Press.

Davis, G. E. 1955. Scattering of light by an air bubble in water. Journal of the Optical Society of America, Vol. 45, No. 7, p. 572-81.

Dunkle, R. V., and Bevans, J. T. 1956. An approximate analysis of the solar reflectance and transmittance of a snow cover. Journal of Meteorology, Vol. 13, No. 2, p. 212-16.

Ellison, J. M., and Peetz, C. U. 1959. The forward scattering of light by spheres according to geometrical optics. Proceedings of the Physical Society of London, Vol. 74, Pt. 1, No. 475, p. 105-22.

Goodrich, L. E. 1970. Review of radiation absorption coefficients for clear ice in the spectral region 0.3 to 3 microns. Canada. National Research Council. Division of Building Research. Technical Paper No. 331.

Grenfell, T. C., and Maykut, G. A. 1977. The optical properties of ice and snow in the Arctic basin. Journal of Glaciology, Vol. 18, No. 80, p. 445-63.

Henyey, L. G., and Greenstein, J. L. 1941. Diffuse radiation in the galaxy. Astrophysical Journal, Vol. 93, No. 1, p. $70-83$.

Jerlov, N. G. 1976. Marine optics. Amsterdam, Elsevier Scientific Publishing Co.

Joseph, J. H., and others. 1976. The delta-Eddington approximation for radiative flux transfer, by J. H. Joseph, W. J. Wiscombe, and J. A. Weinman. Journal of the Atmospheric Sciences, Vol. 33, No. 12, p. $2452-59$.

Liou, K. N. 1973. A numerical experiment on Chandrasekhar's discrete-ordinate method for radiative transfer: applications to cloudy and hazy atmospheres. Journal of the Atmospheric Sciences, Vol. 30, No. 7, p. 1303-26. 
Liou, K. N. 1974. Analytic two-stream and four-stream solutions for radiative transfer. Journal of the Atmospheric Sciences, Vol. 31, No. 5, p. 1473-75.

Papoulis, A. 1965. Probability, random variables, and stochastic processes. New York, McGraw-Hill Book Co., Inc. Perovich, D. K. 1979. The optical properties of young sea ice. Seattle, Dept. of Atmospheric Sciences, University of Washington. (U.S. Office of Naval Research. Contract N00014-76-C-0234. Project No. 307-252. Scientific Report No. 17.)

Perovich, D. K., and Grenfell, T. C. 1981. Laboratory studies of the optical properties of young sea ice. Journal of Glaciology, Vol. 27, No. 96, p. 331-46.

Roulet, R. R., and others. 1974. Spectrophotometers for the measurement of light in polar ice and snow, by R. R. Roulet, G. A. Maykut, and T. C. Grenfell. Applied Optics, Vol. 13, No. 7, p. 1652-58.

Sauberer, F. 1950. Die spektrale Strahlungsdurchlässigkeit des Eises. Wetter und Leben, Jahrg. 2, Ht. 9-10, p. $143-97$. 